\title{
Breeding value accuracy estimates for growth traits using random regression and multi-trait models in Nelore cattle
}

\author{
A.A. Boligon ${ }^{1}$, F. Baldi' ${ }^{1}$ M.E.Z. Mercadante ${ }^{2}$, R.B. Lobo ${ }^{3}$, R.J. Pereira ${ }^{1}$ \\ and L.G. Albuquerque ${ }^{1}$ \\ ${ }^{1}$ Faculdade de Ciências Agrárias e Veterinárias, Universidade Estadual Paulista \\ "Júlio de Mesquita Filho", Jaboticabal, SP, Brasil \\ ${ }^{2}$ Instituto de Zootecnia, Estação Experimental de Zootecnia de Sertãozinho, \\ Sertãozinho, SP, Brasil \\ ${ }^{3}$ Departamento de Genética, Faculdade de Medicina de Ribeirão Preto, \\ Universidade de São Paulo, Ribeirão Preto, SP, Brasil \\ Corresponding author: A.A. Boligon \\ E-mail: arioneboligon@yahoo.com.br
}

Genet. Mol. Res. 10 (2): 1227-1236 (2011)

Received October 1, 2010

Accepted December 10, 2010

Published June 28, 2011

DOI 10.4238/vol10-2gmr1087

\begin{abstract}
We quantified the potential increase in accuracy of expected breeding value for weights of Nelore cattle, from birth to mature age, using multi-trait and random regression models on Legendre polynomials and B-spline functions. A total of 87,712 weight records from 8144 females were used, recorded every three months from birth to mature age from the Nelore Brazil Program. For random regression analyses, all female weight records from birth to eight years of age (data set I) were considered. From this general data set, a subset was created (data set II), which included only nine weight records: at birth, weaning, 365 and 550 days of age, and 2, 3, 4, 5, and 6 years of age. Data set II was analyzed using random regression and multi-trait models. The model of analysis included the contemporary group as fixed effects and age of dam as a linear and quadratic covariable. In
\end{abstract}


the random regression analyses, average growth trends were modeled using a cubic regression on orthogonal polynomials of age. Residual variances were modeled by a step function with five classes. Legendre polynomials of fourth and sixth order were utilized to model the direct genetic and animal permanent environmental effects, respectively, while third-order Legendre polynomials were considered for maternal genetic and maternal permanent environmental effects. Quadratic polynomials were applied to model all random effects in random regression models on B-spline functions. Direct genetic and animal permanent environmental effects were modeled using three segments or five coefficients, and genetic maternal and maternal permanent environmental effects were modeled with one segment or three coefficients in the random regression models on B-spline functions. For both data sets (I and II), animals ranked differently according to expected breeding value obtained by random regression or multi-trait models. With random regression models, the highest gains in accuracy were obtained at ages with a low number of weight records. The results indicate that random regression models provide more accurate expected breeding values than the traditionally finite multi-trait models. Thus, higher genetic responses are expected for beef cattle growth traits by replacing a multi-trait model with random regression models for genetic evaluation. B-spline functions could be applied as an alternative to Legendre polynomials to model covariance functions for weights from birth to mature age.

Key words: B-spline functions; Multi-trait model; Genetic parameters; Legendre polynomials; Random regression models; Rank correlations

\section{INTRODUCTION}

Generally, in beef cattle, breeding values for growth traits are predicted by finite models using multi-trait analyses (MT). For fitting these models, weights are standardized to specific ages and the weights that are out of the accepted age range are discarded from the analyses. Nowadays, random regression models (RRM) have been used to estimate genetic parameters for growth traits in beef cattle. With these models, breeding values are predicted using all records available, in a continuous manner for any age or point in the trajectory, instead of finite dimensional models that only give point predictions of breeding values (Meyer, 2004).

Recently, spline functions have been proposed as alternative functions to orthogonal polynomials to model growth trajectories of beef cattle. Meyer (2005b) reported that RRM fitting B-splines as basis functions were less susceptible to the "end-of-range" problems frequently observed for analyses fitting polynomial regression. Also, in applying spline functions, the computational requirements are less than for corresponding analyses with the same number of coefficients but using Legendre polynomial (LP), since the matrices in the mixed model are sparser with spline functions (Meyer, 2005b; Misztal, 2006).

The accuracy of expected breeding values (EBV), the squared correlation between 
true and estimated breeding value, provides an assessment of risk associated with selection decisions. Accuracy estimates for a trait vary with the amount and structure of the data used to predict the EBV. Boligon et al. (2008), analyzing Nelore cow weight records, showed that larger EBV accuracies were obtained with a repeatability animal model than with a single record model. From simulated data, there is sufficient evidence that RRM provide estimates of breeding values with higher accuracies than conventional finite dimensional models (Meyer, 2004; Bohmanova et al., 2005). However, there are no studies comparing the EBV accuracies obtained by RRM or finite dimensional models using real data. Generally, field weight records are taken at irregular intervals, and, particularly in selection situations, the data are not necessarily missing at random.

Nelore overall growth and mature size have increased over the years as a result of selection for growth traits at early ages (Mercadante et al., 2003) and, as a consequence, also cow mature size (Silva et al., 2000). Considering that the energy required for maintaining the cow herd is the main cost of beef cattle production systems (Ferrell and Jenkins, 1985), increasing cow mature size may not be ideal in a low input environment. In a breeding program it is important to match cow size to environmental conditions and management and feeding practices.

The selection to maintain or to restrict cow size while increasing saleable weight of calves at weaning and at slaughter requires precise and accurate breeding value estimates for weights at different ages. However, there are difficulties in using mature weights in genetic evaluations due to the scarcity of weight records obtained after 2 years of age. Comparing Bsplines with LP, several papers have shown the advantage of B-splines (Meyer et al., 2005b) or not (Boligon et al., 2009, 2010; Baldi et al., 2010) to model weight records at the extreme of the trajectory (later ages). Nevertheless, to date, it is unclear whether EBVs obtained with RRM on B-spline functions are more accurate than RRM on LP.

The objective of this study was to quantify the potential increase in accuracy of EBV for weights of Nelore cattle, from birth to mature age, using MT and RRM on LP or B-spline functions.

\section{MATERIAL AND METHODS}

\section{Data}

Data came from eight herds located in São Paulo State, participating in the Program for Genetic Improvement of the Nelore Breed (Nelore Brazil). Only records of animals that were reared on pasture, without supplemental feed, and weaned by their biological mothers were considered. Animals were born from 1976 to 2000 and were daughters of 530 sires and 2978 cows aged from 2 to 24 years. The births occurred throughout the year, but were more frequent during spring (September to November) and summer (December to February). Animals were weighed every 90 days during their entire herd life. Weaning occurred at 240 days of age, on average.

For random regression analyses, all females' weight records from birth to eight years of age (data set I) were considered. From this general data set, a subset was created (data set II), including only nine weight records: at birth, weaning, 365 and 550 days of age and 2, 3, 4,5 , and 6 years of age. Data set II was analyzed using RRM and the MT model. There were a total of 12,596 animals in the relationship matrix. A statistical summary of data structure is 
presented in Table 1. The distribution of weight records for both data sets is presented in Figure 1. A decrease in the number of animals was observed after 600 days of age, suggesting the occurrence of selection up to 18 months.

\begin{tabular}{lcc} 
Table 1. Summary of data structure for data set considering all weight records available (data set I) and only \\
records at standard ages (data set II). \\
\hline
\end{tabular}

Total number of animals $=12,596 ;$ number of sires $=530 ;$ number of dams $=2978$.

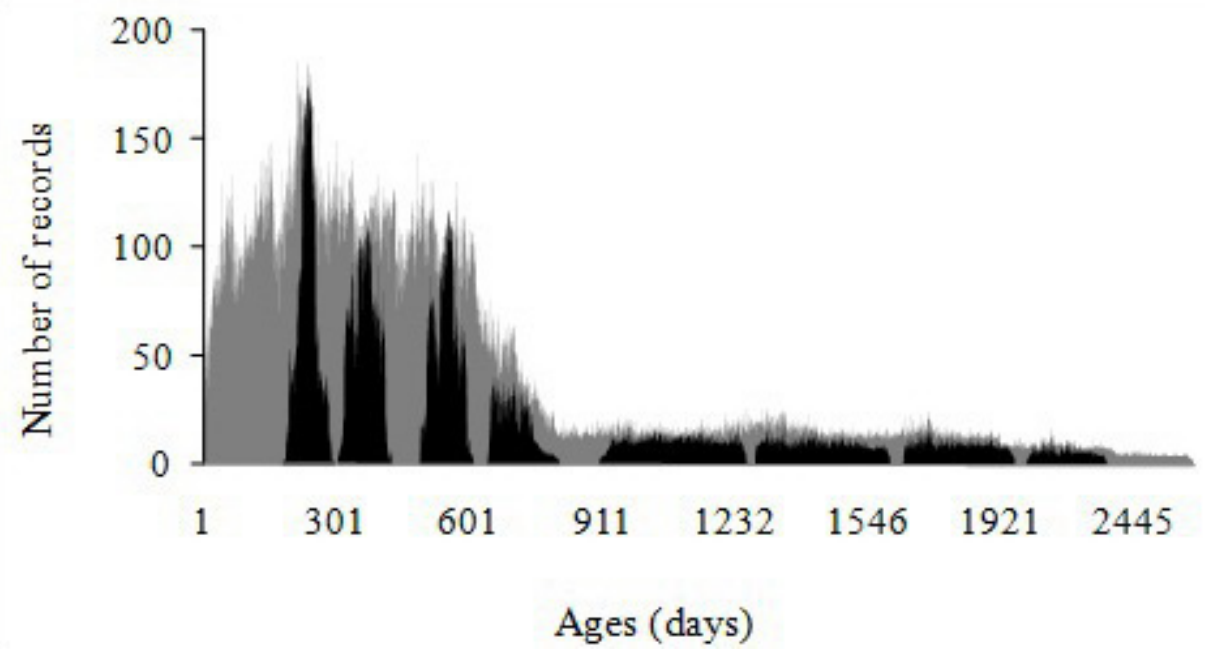

Figure 1. Number of weight records according to age at recording. Data set I (gray) and data set II (black). Birth weight records are not shown.

\section{Analyses}

The EBV and corresponding accuracies were obtained considering the following models: MT, using nine weight records; RRM-LP, RRM on LP of age; RRM-spline, RRM on Bspline functions of age. The MT model included weight records at birth (BW), weaning (W240), 365 (W365) and 550 (W550) days of age, and 2 (W2Y), 3 (W3Y), 4 (W4Y), 5 (W5Y), and 6 (W6Y) years of age. For all weights, direct and maternal additive genetic and animal and maternal permanent environmental effects were considered as random. Contemporary group (CG-MT: herd, year and month of birth) was included in the models as fixed effect. Linear and quadratic effects of animal age at weighing (except for birth weight) and dam age at calving were included as covariables. Correlations between direct additive genetic and maternal genetic effects were set to zero as suggested by Benyshek et al. (1988) and Albuquerque and Meyer (2001). 
In the random regression analyses, mean trends were modeled using a cubic regression on orthogonal polynomials of age. Residual variances were modeled by a step function with 5 classes $(0 ; 1-216 ; 217-660 ; 661-960 ; 961-2889$ days of age). Contemporary groups were defined as GC-MT plus age at recording class (birth and every 45 days of age). For records taken after 3 years of age, CG was defined by farm, year and season of recording. The fixed effects of CG and age of dam as a covariable (linear and quadratic effects) were included in the model. Since animal weight variation depended on the age of the dam, animal age was considered to be within five dam age classes, defined as follows: 2-3, 4-5, 6-8, 9-11, 12-24 years of age.

Legendre polynomials of fourth and sixth orders were used to model the direct genetic and permanent environmental effects, respectively, while a third-order polynomial was used for maternal genetic and permanent environmental effects. In the RRM-spline, random effects were modeled by applying quadratic B-spline functions. The additive direct genetic and permanent environmental effects were modeled with 4 knots ( 2 internal and 2 external), at equidistant intervals of 963 days, corresponding to 3 segments. Maternal genetic and permanent environmental effects were modeled with 2 external knots (only one segment). For more details, see Boligon et al. (2009, 2010).

The covariance components and genetic parameters were estimated by restricted maximum likelihood (REML), using the WOMBAT software (Meyer, 2006). For weights from birth to mature age, Spearman correlations were estimated between sires' breeding values obtained by different models (MT, RRM-LP and RRM-spline).

\section{Accuracy estimates}

The EBV accuracy estimates for each weight in the MT analyses were calculated from prediction error variance (PEV), obtained from the diagonal elements of the inverse of the coefficient matrix in mixed model equations. Selected elements of the inverse of the coefficient matrix, corresponding to the diagonal blocks pertaining to sets of random regression coefficients for additive direct effect, were estimated for RRM-LP and RRM-spline.

In RRM, the diagonal block for each set of random regression coefficients provides estimates of an animal's prediction coefficients and prediction error variances (Meyer, 2004). Once the PEV for the genetic random regression coefficients are obtained, the PEV for specific ages can be obtained by evaluating the estimated regression for the ages desired. While this is a polynomial function of age, it is linear in the estimated RR coefficients. For both, RRM and MT models, the expected accuracy was then calculated as

$$
\sqrt{1-P E V_{i} / \text { Var }_{i}}
$$

(Equation 1)

where $P E V_{i}$ corresponds to the predicted error variance at age $i$ and $V a r_{i}$ is the additive genetic variance for weight at age $i$ (Meyer, 2004).

\section{RESULTS AND DISCUSSION}

In general, using both data sets, the heritability estimates obtained with all the models were very similar. However, heritability estimates for birth weight and weights after 3 years of age obtained by the MT model were slightly lower than those using RRM. For all weights, the heri- 
tability estimates obtained in data set I were slightly higher than those estimated with data set II (results not shown). Discussion about these estimates is presented by Boligon et al. (2009, 2010).

For weights from weaning to mature age, the mean of EBV obtained with RRM-LP and RRM-spline was higher than with the MT model (Table 2). In general, RRM fitted the data better and produced higher heritability estimates compared to MT. However, using weight records from birth to yearling, also in Nelore cattle, Albuquerque and El Faro (2008) reported higher EBV values estimated with standard two-trait analyses compared to RRM.

\begin{tabular}{|c|c|c|c|c|c|c|c|c|c|}
\hline \multirow[t]{3}{*}{ Trait } & \multicolumn{9}{|c|}{ Data set I } \\
\hline & \multicolumn{5}{|c|}{ RRM-LP } & \multicolumn{4}{|c|}{ RRM-spline } \\
\hline & \multicolumn{2}{|c|}{ Mean \pm SD } & Min. & \multicolumn{2}{|l|}{ Max. } & Mean \pm SD & \multicolumn{2}{|c|}{ Min. } & Max. \\
\hline \multicolumn{2}{|l|}{ BW } & $0.38 \pm 1.09$ & -4.15 & \multicolumn{2}{|c|}{5.21} & $0.36 \pm 1.12$ & \multicolumn{2}{|c|}{-4.18} & 5.30 \\
\hline W240 & \multicolumn{2}{|c|}{$1.39 \pm 2.04$} & -7.84 & \multicolumn{2}{|l|}{14.97} & $1.41 \pm 2.09$ & \multicolumn{2}{|c|}{-7.67} & 15.01 \\
\hline W365 & \multicolumn{2}{|c|}{$2.17 \pm 2.57$} & -8.58 & \multicolumn{2}{|l|}{22.54} & $2.11 \pm 2.42$ & \multicolumn{2}{|c|}{-8.33} & 21.89 \\
\hline W550 & \multicolumn{2}{|c|}{$3.98 \pm 3.02$} & -8.22 & \multicolumn{2}{|l|}{33.56} & $3.77 \pm 2.94$ & \multicolumn{2}{|c|}{-7.97} & 32.90 \\
\hline W2Y & \multicolumn{2}{|c|}{$5.28 \pm 6.81$} & -12.27 & \multicolumn{2}{|l|}{41.68} & $5.19 \pm 6.64$ & \multicolumn{2}{|c|}{-12.08} & 41.05 \\
\hline W3Y & \multicolumn{2}{|c|}{$4.34 \pm 5.99$} & -10.18 & \multicolumn{2}{|l|}{39.87} & $4.57 \pm 5.63$ & -9.3 & & 39.24 \\
\hline W4Y & & \pm 12.24 & -22.57 & 43.09 & & $6.88 \pm 11.45$ & -20.8 & & 44.17 \\
\hline W5Y & & \pm 10.26 & -20.09 & 44.28 & & $6.24 \pm 10.31$ & -20.1 & & 44.55 \\
\hline W6Y & & \pm 9.87 & -23.01 & 43.69 & & $6.99 \pm 10.02$ & -23.2 & & 43.57 \\
\hline Trait & & & & & Data set I & & & & \\
\hline & & MT & & & RRM-LP & & & RM-splin & \\
\hline & Mean \pm SD & Min. & Max. & Mean \pm SD & Min. & Max. & Mean \pm SD & Min. & Max. \\
\hline BW & $0.24 \pm 1.09$ & -4.74 & 5.37 & $0.37 \pm 1.24$ & -4.21 & 5.45 & $0.35 \pm 1.10$ & -4.11 & 5.34 \\
\hline W240 & $1.04 \pm 2.19$ & -8.01 & 15.22 & $1.33 \pm 2.05$ & -7.98 & 15.03 & $1.36 \pm 2.11$ & -7.72 & 14.89 \\
\hline W365 & $1.98 \pm 2.82$ & -8.87 & 23.19 & $2.09 \pm 2.37$ & -8.21 & 22.71 & $2.01 \pm 2.39$ & -8.41 & 21.09 \\
\hline W550 & $3.08 \pm 3.27$ & -8.36 & 34.07 & $3.43 \pm 2.78$ & -7.09 & 32.07 & $3.03 \pm 2.65$ & -7.19 & 33.04 \\
\hline W2Y & $3.99 \pm 7.25$ & -11.55 & 42.09 & $5.26 \pm 6.89$ & -12.21 & 42.24 & $5.29 \pm 6.33$ & -12.32 & 40.34 \\
\hline W3Y & $4.20 \pm 6.11$ & -10.58 & 41.32 & $4.37 \pm 5.61$ & -10.22 & 38.12 & $4.30 \pm 5.04$ & -10.01 & 37.38 \\
\hline W4Y & $5.85 \pm 13.08$ & -24.06 & 43.51 & $6.39 \pm 10.87$ & -20.46 & 40.81 & $6.04 \pm 10.70$ & -19.09 & 43.98 \\
\hline W5Y & $4.78 \pm 12.07$ & -24.74 & 46.24 & $5.46 \pm 09.72$ & -19.33 & 40.19 & $5.98 \pm 10.08$ & -20.39 & 40.23 \\
\hline W6Y & $5.04 \pm 11.25$ & -25.41 & 45.03 & $6.08 \pm 9.22$ & -22.43 & 41.07 & $6.51 \pm 09.76$ & -22.54 & 42.69 \\
\hline
\end{tabular}

In the data set considering all weight records available (data set I), the estimates of sire rank correlations between EBVs estimated with both RRM were slightly higher (ranging from 0.90 to 0.98 ) than those obtained with the same models in data set II (ranging from 0.87 to 0.94) (Table 3). For data set II, the sire rank correlations between EBVs obtained by MT and RRM varied from 0.53 to 0.76 . Nevertheless, rank correlations above 0.87 were observed between EBVs obtained by RRM-LP and RRM-spline. These results indicated that practically the same sires would be selected independent of the RRM model applied for genetic evaluation. On the other hand, different sires would be selected if an RRM were used instead of an MT model. Differences in sire classification between MT and RRM are expected since, by applying an MT model, weights are arbitrarily grouped in classes and adjusted to standard ages. With RRM, all weights are considered to be in the analysis and the effect of age is included in the random coefficient matrix (Nobre et al., 2003). Consequently, random and fixed effects are better modeled with RRM (Meyer, 1998). 


\begin{tabular}{|c|c|c|c|c|}
\hline \multirow[t]{2}{*}{ Trait } & \multirow{2}{*}{$\frac{\text { Data set I (530 sires) }}{\text { RRM-LP x RRM-spline }}$} & \multicolumn{3}{|c|}{ Data set II (530 sires) } \\
\hline & & MT x RRM-LP & MT x RRM-spline & RRM-LP x RRM-spline \\
\hline$\overline{\mathrm{BW}}$ & 0.93 & 0.69 & 0.67 & 0.90 \\
\hline W240 & 0.94 & 0.72 & 0.71 & 0.91 \\
\hline W365 & 0.96 & 0.70 & 0.74 & 0.93 \\
\hline W550 & 0.98 & 0.74 & 0.76 & 0.96 \\
\hline W2Y & 0.96 & 0.69 & 0.70 & 0.94 \\
\hline W3Y & 0.95 & 0.62 & 0.63 & 0.92 \\
\hline W4Y & 0.94 & 0.64 & 0.62 & 0.90 \\
\hline W5Y & 0.91 & 0.55 & 0.56 & 0.89 \\
\hline W6Y & 0.90 & 0.53 & 0.50 & 0.87 \\
\hline
\end{tabular}

$\mathrm{BW}=$ birth weight; $\mathrm{W} 240$ = weaning weight; $\mathrm{W} 365$ = weight at 365 days; $\mathrm{W} 550$ = weight at 550 days; $\mathrm{W} 2 \mathrm{Y}, \mathrm{W} 3 \mathrm{Y}$, $\mathrm{W} 4 \mathrm{Y}, \mathrm{W} 5 \mathrm{Y}$, and $\mathrm{W} 6 \mathrm{Y}=$ weight at 2, 3, 4, 5, and 6 years of age, respectively.

In the present study, large differences in sire classification between EBV obtained from MT or RRM were observed for birth weight and for weights after three years of age (Table 3). The small number of records at these ages probably contributed to the differences observed.

Comparing the EBVs of weights from birth to yearling obtained by MT or RRM-LP, Albuquerque and El Faro (2008) reported rank correlations that varied from 0.64 to 0.87 (considering all the animals) and from 0.59 to 0.87 (considering only the sires). Also in Nelore cattle, Nobre et al. (2003) analyzed a large data set including weight records from birth to 733 days of age, reporting less differences in the animal classification (rank correlation varied from 0.80 to 0.89 ) based on EBV obtained by MT or RRM-LP. Sanchez et al. (2008a) working with Gelbvieh cattle, compared the EBV obtained by multi-trait analyses (birth, weaning and yearling weight) or RRM using linear splines and indicated that minor changes were obtained in the overall rank of bulls after the inclusion of extra information from animals outside the normal measurement age range.

In the data set considering weight records measured at standard ages (data set II) the accuracies of EBV obtained by RRM were slightly higher than those estimated with the MT model (Table 4). This could be attributed to more appropriate modeling of variances and genetic parameters with the RRM (Boligon et al., 2010). As would expected, by including all the available weight records in the analyses (data set I), considerably higher accuracies were obtained with RRM, and these differences were larger for ages with small number of records (up to 67\%), such as birth and weights measured at later ages (Table 4). Thus, RRMs resulted in more accurate EBVs due to the inclusion of all weight records available and better modeling of the covariance structure of the data. Meyer (2004), with simulated data, also reported higher accuracies using RRM instead of MT, but EBV accuracies obtained with RRM were only slightly higher than those with MT (up to 8.3\%). Bohmanova et al. (2005), also working with simulated data and weight records measured at standard ages (birth, 205 and 365 days of age), showed that EBV accuracy estimates obtained by MT and RRM were similar (using LP or spline functions). However, considering weight records measured randomly along ages, RRM showed slightly higher EBV accuracy estimates than the MT model. The results obtained in the present study confirm that EBVs obtained with RRM are more reliable than those estimated with finite dimensional models as already demonstrated in simulated studies. However, the advantage of RRM over finite dimensional models was less evident with simulated data than with real data. With simulated data, results are probably highly dependent on assumptions considered for both genetic parameter estimates and data structure. 


\begin{tabular}{|c|c|c|c|c|c|c|c|c|c|}
\hline \multirow[t]{3}{*}{ Trait } & & \multicolumn{8}{|c|}{ Data set I (530 sires) } \\
\hline & & \multicolumn{4}{|c|}{ RRM-LP } & \multicolumn{4}{|c|}{ RRM-spline } \\
\hline & & Mean & Min. & \multicolumn{2}{|c|}{ Max. } & Mean & \multicolumn{2}{|c|}{ Min. } & Max. \\
\hline BW & & 0.75 & 0.67 & \multicolumn{2}{|c|}{0.89} & 0.79 & \multicolumn{2}{|c|}{0.68} & 0.89 \\
\hline W240 & & 0.82 & 0.72 & \multicolumn{2}{|c|}{0.96} & 0.84 & \multicolumn{2}{|c|}{0.74} & 0.94 \\
\hline W365 & & 0.80 & 0.69 & \multicolumn{2}{|c|}{0.91} & 0.82 & \multicolumn{2}{|c|}{0.71} & 0.93 \\
\hline W550 & & 0.76 & 0.65 & \multicolumn{2}{|c|}{0.93} & 0.78 & \multicolumn{2}{|c|}{0.67} & 0.92 \\
\hline W2Y & & 0.72 & 0.67 & \multicolumn{2}{|c|}{0.92} & 0.73 & \multicolumn{2}{|c|}{0.66} & 0.94 \\
\hline W3Y & & 0.71 & 0.64 & \multicolumn{2}{|c|}{0.94} & 0.72 & \multicolumn{2}{|c|}{0.67} & 0.95 \\
\hline W4Y & & 0.80 & 0.73 & \multicolumn{2}{|c|}{0.95} & 0.82 & \multicolumn{2}{|c|}{0.72} & 0.94 \\
\hline W5Y & & 0.80 & 0.68 & \multicolumn{2}{|c|}{0.92} & 0.83 & \multicolumn{2}{|c|}{0.70} & 0.99 \\
\hline W6Y & & 0.78 & 0.64 & \multicolumn{2}{|c|}{0.90} & 0.81 & \multicolumn{2}{|c|}{0.68} & 0.96 \\
\hline \multirow[t]{3}{*}{ Trait } & \multicolumn{9}{|c|}{ Data set II (530 sires) } \\
\hline & \multicolumn{3}{|c|}{ MT } & \multicolumn{3}{|c|}{ RRM-LP } & \multicolumn{3}{|c|}{ RRM-spline } \\
\hline & Mean & Min. & Max. & Mean & Min. & Max. & Mean & Min. & Max. \\
\hline BW & 0.51 & 0.30 & 0.71 & 0.57 & 0.44 & 0.70 & 0.61 & 0.50 & 0.78 \\
\hline W240 & 0.59 & 0.37 & 0.81 & 0.64 & 0.54 & 0.81 & 0.67 & 0.56 & 0.79 \\
\hline W365 & 0.58 & 0.35 & 0.76 & 0.62 & 0.53 & 0.76 & 0.64 & 0.56 & 0.78 \\
\hline W550 & 0.54 & 0.38 & 0.78 & 0.58 & 0.48 & 0.77 & 0.59 & 0.48 & 0.79 \\
\hline W2Y & 0.49 & 0.27 & 0.76 & 0.53 & 0.46 & 0.72 & 0.54 & 0.47 & 0.75 \\
\hline W3Y & 0.47 & 0.24 & 0.74 & 0.52 & 0.44 & 0.71 & 0.53 & 0.49 & 0.70 \\
\hline W4Y & 0.54 & 0.22 & 0.76 & 0.60 & 0.51 & 0.77 & 0.62 & 0.53 & 0.78 \\
\hline W5Y & 0.52 & 0.27 & 0.79 & 0.60 & 0.50 & 0.79 & 0.62 & 0.53 & 0.81 \\
\hline W6Y & 0.48 & 0.25 & 0.75 & 0.59 & 0.46 & 0.74 & 0.62 & 0.46 & 0.76 \\
\hline
\end{tabular}

$\mathrm{BW}=$ birth weight; $\mathrm{W} 240$ = weaning weight; $\mathrm{W} 365$ = weight at 365 days; $\mathrm{W} 550=$ weight at 550 days; W2Y, W3Y, $\mathrm{W} 4 \mathrm{Y}, \mathrm{W} 5 \mathrm{Y}$, and $\mathrm{W} 6 \mathrm{Y}=$ weight at $2,3,4,5$, and 6 years of age, respectively.

For both data sets, the EBV accuracies obtained with RRM-spline were equal or higher than those estimated by RRM-LP (Table 4), particularly at extreme ages where RRM-spline accuracies were from 5 to $15 \%$ higher with respect to RRM-LP. This fact suggests that for ages with small number of records, RRM-spline fits the data better than RRM-LP. For Meyer (2005a), B-spline functions were less susceptible to estimation problems at the end of the trajectory, due to the lower degree of polynomials fitted in the individual segments. Our results differ from those reported by Bohmanova et al. (2005) and Sanchez et al. (2008b). The first authors used simulated weights, from birth to 410 days of age, and reported slightly more accurate EBVs with RRM-LP than with RRM-spline. Sanchez et al. (2008b), working with Gelbvieh weight data (birth, weaning and yearling weight), reported similar EBV accuracy estimates obtained by RRM on LP or spline functions.

The genetic gain in an animal breeding program depends on, among other factors, the availability of accurate EBVs for young candidates for selection. Usually, EBVs for growth traits of young sires (with no or just a few offspring) have shown low accuracies. In the current study, accuracy estimates of EBVs for young sires without progeny records varied from 0.76 to 0.79 with RRM (data set I) and from 0.61 to 0.66 when considering the MT model (data set II). The results obtained in the present study are even more important for young bulls with no or just a few progeny. These conclusions agreed with those drawn by Boligon et al. (2008), who showed that EBV accuracy estimates of young candidates (with no progeny information) for mature cow weight changed from $0.39-0.44$ when considering single records to $0.64-0.76$ when considering repeated records. Therefore, when weight records were analyzed by RRM instead of MT, it was 
possible to change the EBV accuracies from low to moderate, thus increasing genetic gain.

Currently, Brazilian animal breeding programs are concerned with the increase in mature cow size, since growth traits are included as selection criteria in all of these programs. As demonstrated in this study, considerable differences in genetic values and gains in accuracy of female weights at older ages were observed when using RRM in relation to the MT model, indicating that the use of RRM in genetic evaluation of beef cattle tends to be more advantageous for weights that generally have a smaller number of measures, such as mature weight.

The results of this study show that in circumstances in which there are enough weight records at standard ages, the results obtained with MT and RRM are expected to be equivalent. Otherwise, when weight records are not equally distributed at specific ages, RRM showed more accurate EBV than with MT models. With RRM, accuracy estimates tend to increase as more records are incorporated in the analyses.

EBV accuracy for sires obtained with RRM-spline was slightly higher than with RRM-LP, suggesting that B-spline functions could be applied as an alternative to LP to model covariance functions for weights from birth to adult weight. Meyer (2005b) suggested that $\mathrm{B}$-spline functions are more suitable for describing the covariance structure of the data at the extreme of the trajectory compared to LP. The better properties of B-spline functions probably explain the differences obtained with LP.

\section{CONCLUSIONS}

The results indicate that random regression models provide more accurate EBVs over the traditionally finite multi-trait models. Thus, higher genetic gains are expected for beef cattle growth traits by replacing a multi-trait model with random regression models for genetic evaluation. B-spline functions could be applied as an alternative to Legendre polynomials to model covariance functions for weights from birth to mature age.

\section{ACKNOWLEDGMENTS}

Research supported by Fundação de Amparo à Pesquisa do Estado de São Paulo (FAPESP). We are indebted to the Associação Nacional de Criadores e Pesquisadores (ANCP) for the use of the dataset.

\section{REFERENCES}

Albuquerque LG and Meyer K (2001). Estimates of direct and maternal genetics effects for weights from birth to 600 days of age in Nelore cattle. J. Anim. Breed. Genet. 118: 83-92.

Albuquerque LG and El Faro L (2008). Comparações entre os valores genéticos para características de crescimento de bovinos da raça Nelore preditos com modelos de dimensão finita ou infinita. Rev. Bras. Zootec. 37: 238-246.

Baldi F, Alencar MM and Albuquerque LG (2010). Random regression analyses using B-splines functions to model growth from birth to adult age in Canchim cattle. J. Anim. Breed. Genet. 127: 433-441.

Benyshek LL, Johnson MH, Little DE, Bertrand JK, et al. (1988). Application of an animal model in the United States beef cattle industry. J. Dairy Sci. 71: 35-53.

Bohmanova J, Misztal I and Bertrand JK (2005). Studies on multiple trait and random regression models for genetic evaluation of beef cattle for growth. J. Anim. Sci. 83: 62-67.

Boligon AA, Albuquerque LG, Mercadante ME and Lobo RB (2008). Models for genetic evaluation of Nelore cattle mature body weight. J. Anim. Sci. 86: 2840-2844. 
Boligon AA, Albuquerque LG, Mercadante MEZ and Lobo RB (2009). Estimates of genetic parameters using random regression on B-spline functions for weights from birth to mature in Nellore cattle. In: 2009 JOINT ADSA ${ }^{\circledR}$-CSASASAS MEETING, 2009, Montreal, Quebec, Canada.

Boligon AA, Mercadante ME, Forni S, Lobo RB, et al. (2010). Covariance functions for body weight from birth to maturity in Nellore cows. J. Anim. Sci. 88: 849-859.

Ferrell CL and Jenkins TG (1985). Cow type and the nutritional environment: nutritional aspects. J. Anim. Sci. 61: 725-741.

Mercadante ME, Packer IU, Razook AG, Cyrillo JN, et al. (2003). Direct and correlated responses to selection for yearling weight on reproductive performance of Nelore cows. J. Anim. Sci. 81: 376-384.

Meyer K (1998). Estimating covariance functions for longitudinal data using a random regression model. Genet. Sel. Evol. 30: $221-240$.

Meyer K (2004). Scope for a random regression model in genetic evaluation of beef cattle for growth. Livest. Prod. Sci. 86: 69-83.

Meyer K (2005a). Estimates of genetic covariance functions for growth of Angus cattle. J. Anim. Breed. Genet. 122: 73-85.

Meyer K (2005b). Random regression analyses using B-splines to model growth of Australian Angus cattle. Genet. Sel. Evol. 37: 473-500.

Meyer K (2006). "WOMBAT" Digging Deep for Quantitative Genetic Analyses by Restricted Maximum Likelihood. Proceedings of the 8th World Congress on Genetics Applied to Livestock Production, CD-ROOM, Belo Horizonte.

Misztal I (2006). Properties of random regression models using linear splines. J. Anim. Breed. Genet. 123: 74-80.

Nobre PR, Misztal I, Tsuruta S, Bertrand JK, et al. (2003). Genetic evaluation of growth in nellore cattle by multiple-trait and random regression models. J. Anim. Sci. 81: 927-932.

Sanchez JP, Misztal I, Aguilar I and Bertrand JK (2008a). Genetic evaluation of growth in a multibreed beef cattle population using random regression-linear spline models. J. Anim. Sci. 86: 267-277.

Sanchez JP, Misztal I and Bertrand JK (2008b). Evaluation of methods for computing approximate accuracies of predicted breeding values in maternal random regression models for growth traits in beef cattle. J. Anim. Sci. 86: 1057-1066.

Silva AM, Alencar MM, Freitas AR, Barbosa RT, et al. (2000). Herdabilidade e correlações genéticas para peso e perímetro escrotal de machos e características reprodutivas e de crescimento de fêmeas, na raça Canchim. Rev. Bras. Zootec. 29: 2223-2230. 